\title{
High-fat snacks and increased risk of high body-fat gain in school-aged children
}

\author{
T. A. McCaffrey ${ }^{1}$, K. L. Rennie ${ }^{1,2}$, M. A. Kerr ${ }^{1}$, J. M. Wallace ${ }^{1}$, S. A. Jebb ${ }^{3}$ and M. B. E. Livingstone ${ }^{1}$ \\ ${ }^{1}$ Northern Ireland Centre For Food and Health (NICHE), University of Ulster, Coleraine BT52 1SA, UK, ${ }^{2}$ Present address: \\ Unilever Corporate Research, Colworth House, Sharnbrook, Bedfordshire MK44 1LQ, UK and ${ }^{3}$ MRC Human Nutrition \\ Research, Elsie Widdowson Laboratory, Fulbourn Road, Cambridge CB1 9NL, UK
}

Maintaining a healthy body weight has now become a major challenge in the face of an increasingly obesogenic environment. The relevance of snacking patterns in the aetiology of excess adiposity gain in children remains equivocal ${ }^{(1-3)}$. Prospective relationships between snacking patterns and change in body fatness were investigated in forty-eight children (thirty boys and eighteen girls), who were initially studied at 6-8 years of age (baseline; BL) and followed up at 13-17 years of age (FU). At BL energy intake (EI) was assessed by $7 \mathrm{~d}$ weighed-food records concurrent with estimates of total energy expenditure (TEE) by doubly-labelled water (DLW) to quantify misreporting (EI:TEE). Eating occasions (meals or snacks) were self-defined by subjects and/or parents. Snacking occasions were categorised based on the average percentage energy from fat; 'low-fat snacking group' (LFSG; <35\% energy from fat for snacks) or 'high-fat snacking group' (HFSG; > 35\% energy from fat for snacks). Body fatness was measured by total body water from DLW (expressed as fat mass index, (FMI; $\mathrm{kg} / \mathrm{m}^{2}$ and \% body fat) and by surrogate measures of body fatness (BMI z-score and waist circumference (WC) $\mathrm{z}$-scores). Change $(\Delta)$ in body fatness was calculated as FU-BL and was categorised based on gender-specific tertiles (top tertile $v$. lower tertiles).

\begin{tabular}{llllrr}
\hline & \multicolumn{2}{c}{ LFSG $(n$ 18) } & & \multicolumn{2}{c}{ HFSG $(n$ 30) } \\
\cline { 2 - 3 } & Median & IQR & & Median & IQR \\
\hline \% EI from snacks & 24.8 & $22.6,32.2$ & & 23.7 & $19.5,30.8$ \\
\% Energy from carbohydrate & 59.9 & $57.3,62.0$ & & 50.4 & $43.7,51.7$ \\
\% Energy from fat & 31.1 & $27.7,32.7$ & & $<0.1$ & $37.3,42.8$ \\
\% Energy from protein & 8.91 & $7.54,11.3$ & 9.83 & $8.12,10.7$ \\
Snack weight (g/d) & 405 & 351,504 & 348 & 259,432 \\
No. of snacking occasions per d & 2.64 & $1.79,3.57$ & 2.00 & 0.624 \\
EI:TEE & 95.5 & $82.1,1.12$ & 96.3 & 0.001 \\
\hline
\end{tabular}

IQR, interquartile range.

There were no significant differences in \% EI from snacks, frequency of snacking occasions or EI:TEE between the LFSG and HFSG $(P>0.05)$. By definition subjects in the HFSG had significantly higher total fat compared with those in the LFSG. Subjects in the HFSG in childhood were eight times more likely to be in the top tertile for fat mass gain ( $\triangle \mathrm{FMI})$ as adolescents, compared with subjects in the LFSG (OR 8.19 (95\% CI 1.33, 50.47); $P=0.023)$. There were no prospective associations between HFSG and $\Delta \%$ body fat or surrogate measures of change in body fatness ( $\triangle \mathrm{BMI}$ z-score and $\Delta \mathrm{WC}$ z-scores). The study highlights a clear association between high-fat snacks and an increased risk of subsequent adiposity (expressed as $\Delta \mathrm{FMI}$ ). However, the associations are dependent on the method used to assess body fatness. These analyses focus particular attention on the nutrient composition of snacks in the diet rather than the 'snacking meal pattern'. These results suggest that public health messages should focus on the nutritional content of snacks, specifically highlighting the advantages of low-fat choices for healthy growth.

The follow up study was commissioned by the Food Standards Agency, UK.

1. Huang TTK, Howarth NC, Lin B-H, Roberts SB \& McCrory MA (2004) Obes Res 12, 1875-1885.

2. Field AE, Austin SB, Gillman MW, Rosner B, Rockett HR \& Colditz GA (2004) Int J Obes Relat Metab Disord 28, $1210-1216$.

3. Ritchie LD, Spector P, Stevens MJ, Schmidt MM, Schreiber GB, Striegel-Moore RH, Wang MC \& Crawford PB (2007) J Nutr 137, 399-406. 